\title{
Virtual Metrology Modeling of Time-Dependent Spectroscopic Signals by a Fused Lasso Algorithm
}

\author{
Chanhee Park ${ }^{\mathrm{a}}$ and Seoung Bum Kim ${ }^{\mathrm{a}, \mathrm{b}, *}$ \\ ${ }^{a}$ School of Industrial Management Engineering \\ Korea University \\ 145 Anam-Ro, Seongbuk-Gu \\ Seoul 136-713, Republic of Korea \\ \{high1212,sbkim1\}@korea.ac.kr \\ Tel: 82-2-3290-3397, Fax: 82-2-929-5888 \\ ${ }^{\mathrm{b}}$ Center for Discrete Mathematics and Theoretical Computer Science, \\ Rutgers, The State University of New Jersey \\ Piscataway, NJ 08854, USA \\ *Corresponding author
}

\begin{abstract}
This paper proposes a fused lasso model to identify significant features in the spectroscopic signals obtained from a semiconductor manufacturing process, and to construct a reliable virtual metrology (VM) model. Analysis of spectroscopic signals involves combinations of multiple samples collected over time, each with a vast number of highly correlated features. This leads to enormous amounts of data, which is a challenge even for modern-day computers to handle. To simplify such complex spectroscopic signals, dimension reduction is critical. The fused lasso is a regularized regression method that performs automatic variable selection for the predictive modeling of highly correlated datasets such as those of spectroscopic signals. Furthermore, the fused lasso is especially useful for analyzing highdimensional data in which the features exhibit a natural order, as is the case in spectroscopic signals. In this paper, we conducted an experimental study to demonstrate the usefulness of a fused lasso-based VM model and compared it with other VM models based on the lasso and
\end{abstract}


elastic-net models. The results showed that the VM model constructed with features selected by the fused lasso algorithm yields more accurate and robust predictions than the lasso- and elastic net-based VM models. To the best our knowledge, ours is the first attempt to apply a fused lasso to VM modeling.

Keywords: fused lasso, feature selection, predictive model, plasma etch, spectroscopic signal, virtual metrology

\section{Introduction}

In semiconductor manufacturing, metrology is required to verify the quality of wafers after the completion of a certain process. As the device dimensions continue to decrease, the lot-tolot process control is being increasingly replaced with the wafer-to-wafer control to achieve tighter process control [1]. However, wafer-to-wafer control requires metrology measurements from every wafer; this leads to an increase in the cost and in the production cycle time. To address these shortcomings, the concept of virtual metrology (VM; Fig. 1) has been developed in the recent years [2]. The metrology measurements should be related to the process conditions. A number of tool process variables that reflect these conditions, such as power, temperature, and flow rate, can be collected from the sensors attached to the tools. The quality variable (e.g., damage, depth, and uniformity) can be measured from the sampled wafers using the metrology equipment. From the relationship between the process variables and the quality variable, a VM model can be constructed to predict the outcomes of the quality variables from the unsampled wafers. Therefore, VM can be used for developing wafer-to-wafer control economically. 


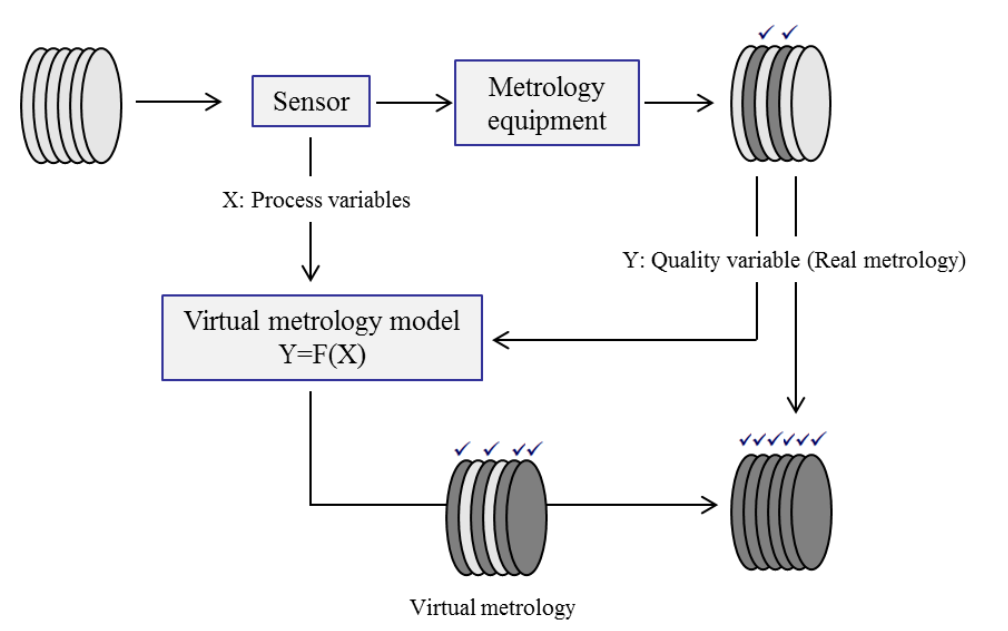

Fig. 1. Conceptual framework of virtual metrology.

A typical semiconductor manufacturing process often involves several hundred operations among which, plasma etching has been recognized as one of the critical operations that directly affects the quality of a wafer [3]. Fig. 2 shows the plasma optical emission intensities that represent the plasma state of a wafer during the etching process. Spectroscopic signals usually consist of thousands of wavelengths, measured over hundreds of time points. This leads to a huge number of features per wafer - a situation that challenges analytical and computational capabilities. In some cases, there may be more observations than features in VM modeling problems. However, in spectroscopic signal used in this study, the number of features exceeds the number of observations in most cases $[21,22,25]$. To address this highdimensionality problem, dimension reduction is critical. In other words, identification of important features that are most predictive of a given target value (metrology measurement of a wafer) is necessary. Furthermore, adjacent features are highly correlated with each other because of the natural sequence present in the signal. In this sense, VM modeling of spectroscopic signals can be challenging [4]. 


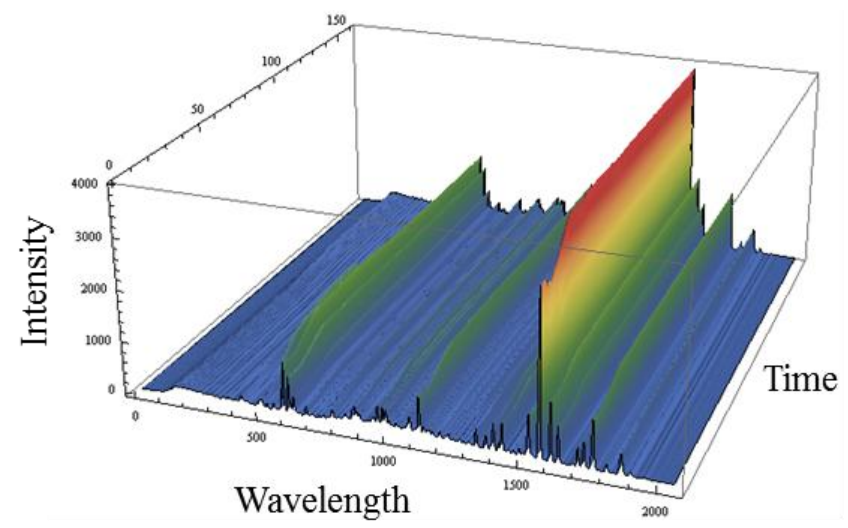

Fig. 2. Time-dependent spectroscopic signals.

To achieve high prediction accuracy for VM, selecting a near-optimal set of features of the process data that can capture the actual characteristics of the product quality is crucial [5]. If we select too many features, irrelevant ones such as noise may be included and they may adversely affect the prediction accuracy of VM [6]. However, selecting too few features may prevent us from capturing important structures of the data [7]. Hence, the identification of the best subset of the features is important for not only developing an accurate model but also facilitating its interpretation. We consider interpretability to be an important issue because engineers who actually operate the semiconductor tools prefer a model that can be easily understood.

Principal component regression (PCR) and partial least squares (PLS) are common regression methods for extracting significant features in a high-dimensional dataset. Both PCR and PLS extract new features by transforming the original features. Generally, the first few features extracted by PCR or PLS are sufficient to satisfy the objective of an analysis; hence, these methods have been widely applied in the VM modeling of high-dimensional data such as spectroscopic signals. Wan et al. investigated a Gaussian process regression-based VM model for predicting etch rate using spectroscopic signals [9]. Prior to training the VM model, they mapped the large input space to a reduced latent feature space using PLS. Park et al. built a PCR-based VM model for etching-rate prediction using plasma information 
parameters [10]. Hirai et al. developed a VM model using locally weighted PLS to predict the etching conversion differential of a plasma etching process [11]. Liu proposed VM model that combined the sparse PLS and the variable importance in projection method (VIP) and showed that their proposed model outperformed the PLS-based approaches [12]. Wang et al. provided a comparison study on feature selection methods for PLS-based VM model. Their experimental results showed that the PLS with VIP model performed the best or the second best for the most of cases [13]. Shang et al. introduced the dynamic PLS-based VM model, which is robust from the temporal noises in process [14]. The simulated examples illustrated that the dynamic PLS-based model performed better than the PLS-based model. Although the PCR and PLS are widely used and robust methods for extracting significant features, but the transformed features obtained from PCR or PLS do not provide a clear interpretation, when compared to the original features, because they are linear combinations of a large number of original features.

Regularized least-square methods provide the best subset of features by solving an optimization problem. Regularized methods are particularly useful for situations in which the number of features exceeds the number of observations that have been frequently encountered in VM modeling. Schirru et al. proposed a VM model based on a ridge regression method to derive a function to characterize the relationship between the process variables and the measurement data [15]. They used the kernel trick to manage the highdimensional process data and extended it to the multilevel kernel ridge regression. The experiment results indicated that their proposed methodology was validated on actual process and measurement data obtained from the semiconductor manufacturing. The lasso [16] is a well-known regularized least-square method that provides a clear interpretation by selecting a subset of features. Pampuri et al. built a VM model based on the lasso to identify meaningful variables in high-dimensional data [17]. The properties of the lasso are extended to a 
hierarchical framework to deal with the data heterogeneity caused by inhomogeneous production and equipment logistics. The experimental results showed that their proposed method was validated on a benchmark dataset from semiconductor manufacturing. Although the lasso has appealing quality for VM modeling, it tends to select only one feature from a group of features that are correlated as in the case of spectroscopic signals. To address this limitation, the elastic net [18] was developed to reflect the grouping tendency to select correlated features. Susto et al. built the VM model based on the elastic net for the early stage measurement of critical production variables [19]. The elastic net-based VM model achieved substantially lower average mean squared error than the lasso-based model. In this study, we propose to use a fused lasso [20] model to construct a reliable VM model. Fused lasso, which is a generalization of the lasso, addresses not only the group effect but also situations in which the features possess some sort of natural order. The characteristic of the fused lasso, which strengthens the grouping tendency so that equal coefficients are assigned to highly correlated and adjacent features, is crucial in enabling a VM model of the spectroscopic signals. To the best of our knowledge, this study is the first to apply a fused lasso model that properly accommodates the characteristics of spectroscopic signals from a semiconductor process.

The spectroscopic signals consist of two factors — wavelength and time. However, previous studies have focused on reduced datasets summarized by their statistical measures such as mean, variance, maximum, and minimum $[9,11,21]$. Because plasma processing is a dynamic process, preserving time information is crucial [22]. In addition, utilization of the statistical measures causes problems related to model interpretability. When only statistical measures are used for constructing a VM model, tracing back to the individual wavelength measurements at a certain point of time is difficult and cumbersome. In this study we adapted the feature-selection procedure for handling the two factors (wavelength and time). The 
proposed procedure provides subsets of selected features that stand for the wavelength and time that are corresponding to the original spectroscopic signals.

The remainder of this paper is organized as follows: Section 2 describes the existing regularized least-squares methods applied in VM. In Section 3, we propose a feature selection procedure for constructing a VM model. Section 4 describes the results of a performance comparison between the proposed method and other regularized least-squares methods using an actual case study. Finally, Section 5 presents our concluding remarks.

\section{Fused lasso and other regularized least-squares methods applied in VM modeling}

In this section, we detail the regularized least-squares methods. VM can be considered as a linear regression problem: given $p$ features $x_{1}, x_{2} \ldots, x_{p}$, the target value $y$ is predicted by

$$
\hat{y}=\hat{\beta}_{0}+x_{1} \hat{\beta}_{1}+\cdots+x_{p} \hat{\beta}_{p}
$$

VM seeks to provide an estimate of $y$ (a metrology measurement) from $x$ (process variable). The vector of coefficients $(\hat{\beta})$ is estimated by the model fitting procedure. The ordinary least-squares (OLS) estimates are obtained by minimizing the residual sum of squares:

$$
\hat{\beta}=\operatorname{argmin}_{\beta} \sum_{i}\left(y_{i}-\sum_{j} x_{i j} \beta_{j}\right)^{2}
$$

It is well known that the OLS solution (2) yields unsatisfactory prediction results in the case of high-dimensional data. Because it overfits the solution on the training dataset by exploiting the degrees of freedom given by the high dimensionality, the prediction of performance of new observations is poor [19]. Regularization methods have been developed to improve OLS. These methods penalize the coefficients to increase the generalization capability, which enhances the prediction of performance of new observations. Ridge regression and lasso are 
the most popular regularized methods used in VM modeling $[17,30]$. For a dataset with $p$ input features, the coefficients for the ridge-regression model are defined as follows:

$$
\hat{\beta}=\operatorname{argmin}_{\beta} \sum_{i}\left(y_{i}-\sum_{j} x_{i j} \beta_{j}\right)^{2}+\lambda \sum_{j=1}^{p} \beta_{j}^{2}
$$

where $\lambda \sum_{j=1}^{p} \beta_{j}{ }^{2}$ is the $L_{2}$-penalty on the regression coefficients and $\lambda$ is the non-negative penalty parameter that controls the amount of shrinkage [24]. Ridge regression achieves a better prediction performance by shrinking coefficients continuously. However, because none of the coefficients are set to zero, the model is not easily interpretable.

The coefficients for the lasso model are calculated as follows:

$$
\hat{\beta}=\operatorname{argmin}_{\beta} \sum_{i}\left(y_{i}-\sum_{j} x_{i j} \beta_{j}\right)^{2}+\lambda \sum_{j=1}^{p}\left|\beta_{j}\right|
$$

where $\lambda \sum_{j=1}^{p}\left|\beta_{j}\right|$ is the $L_{l}$-penalty, which enables the lasso model to provide a sparse solution, because it selects the important features and discards the rest. In comparison with the ridge-regression model, the lasso model is easier to interpret; hence, it is much more appealing for high-dimensional regression problems [16]. However, the lasso model is inappropriate in the following two scenarios [18].

(a) In the case where $p>n$, the lasso selects at most $n$ features before it saturates.

(b) If a group of highly correlated features exists, then the lasso selects only one feature from the group regardless of the importance of the other features.

Thus, the lasso is not ideal for dealing with spectroscopic signals that consist of thousands of features highly correlated with each other, and a small number of observations.

As mentioned before, the elastic net was proposed to overcome the limitations of the lasso. The coefficients of the elastic-net model are defined as follows:

$$
\hat{\beta}=\operatorname{argmin}_{\beta} \sum_{i}\left(y_{i}-\sum_{j} x_{i j} \beta_{j}\right)^{2}+\lambda_{1} \sum_{j=1}^{p}\left|\beta_{j}\right|+\lambda_{2} \sum_{j=1}^{p} \beta_{j}^{2},
$$


where $\lambda_{1}$ and $\lambda_{2}$ are the non-negative penalty parameters, and $\lambda_{1} \sum_{j=1}^{p}\left|\beta_{j}\right|+\lambda_{2} \sum_{j=1}^{p} \beta_{j}^{2}$ is the penalty term for elastic net that combines the lasso and the ridge penalty. When the features are highly correlated, the ridge regression gives them almost the same coefficients. Generally, the elastic net creates a useful compromise between ridge and lasso, which means that strongly correlated features tend to be included in or excluded from the model together [24]. This property helps the elastic net to compensate for the limitation of the lasso in scenarios (a) and (b). The elastic net has previously been applied to VM models of spectroscopic signals in semiconductor manufacturing [15]. This study demonstrated that a VM model with the elastic net outperformed a model with the lasso algorithm in terms of prediction accuracy.

The elastic net appears to be appropriate for constructing a VM model of spectroscopic signals. However, it does not consider the sequence of features, which is one of the natural properties of such signals. In this paper, we propose to use the fused lasso model to select important features for a VM model of spectroscopic signals. The fused lasso algorithm is designed for sparse linear modeling especially when consecutive features are correlated. The coefficients of the fused lasso model are defined as follows:

$$
\hat{\beta}=\operatorname{argmin}_{\beta} \sum_{i}\left(y_{i}-\sum_{j} x_{i j} \beta_{j}\right)^{2}+\lambda_{1} \sum_{j=1}^{p}\left|\beta_{j}\right|+\lambda_{2} \sum_{j=1}^{p}\left|\beta_{j}-\beta_{j-1}\right|,
$$

where $\lambda_{1}$ and $\lambda_{2}$ are the non-negative penalty parameters, and $\sum_{j=1}^{p}\left|\beta_{j}\right|+\sum_{j=1}^{p}\left|\beta_{j}-\beta_{j-1}\right|$ is the fused lasso penalty. The fused lasso algorithm identifies a set of significant features, while removing redundant features which can be considered as noise. The fused lasso can decrease the noise in the data by penalizing differences of neighboring coefficients [29]. In other words, the second penalty term encourages the grouping tendency of adjacent and highly correlated features to be in or out of the model together. This enables the fused lassobased VM model to consider the successive multicollinearity of spectroscopic signals. In 
addition, it overcomes the drawback of the lasso that it selects at most $n$ features in the case where $p>n$.

\section{Feature selection procedure for VM model}

The spectroscopic signals are measurements of the plasma optical emission intensities as a function of time and plasma wavelength. Owing to the large amounts of data, including over 320,000 measurements per wafer, previous studies compressed each wavelength into statistical moments (mean and variance) [3, 4, 25, 26]. However, as plasma etching is a dynamic process depending on time, preserving the original time-domain information is essential. To deal with the enormous number of features and retain the time information, we divided the feature selection procedure into two steps: the first step for determining the wavelengths that are most predictive of a given target value, and the second for selecting the

important time points of the wavelengths extracted in the first step. Fig. 3 shows the pseudocode of the proposed feature-selection procedure for the VM model. 


\footnotetext{
Step 1: Wavelength selection

$n \leftarrow$ Number of observations.

$p \leftarrow$ Number of wavelengths.

$q \leftarrow$ Number of time samples.

$x_{i(j k)} \leftarrow$ Spectroscopic signals, where $1<i<n, 1<j<p, 1<k<q$.

$R \leftarrow$ Penalty term of regularized method.

$y_{i} \leftarrow$ Target values, where $1<i<n$.
}

Each wavelength is summarized by its mean value.

Create reduced dataset from the mean value.

$$
x_{i j}^{\prime} \leftarrow \frac{1}{q} \sum_{k=1}^{q} x_{i(j k)}, \quad \text { for all } i \text { and } j .
$$

Compute the coefficients from the reduced dataset using a regularized method.

$$
\hat{\beta}_{j} \leftarrow \operatorname{argmin}_{\beta} \sum_{i}\left(y_{i}-\sum_{j} x_{i j}^{\prime} \beta_{j}\right)^{2}+R
$$

Select wavelengths with nonzero coefficients.

$$
\text { If } \hat{\beta}_{j}=0: x_{i(j k)} \leftarrow 0 \text {, for all } i, j, k \text {. }
$$

Step 2: Time point selection

Compute the coefficients from the selected dataset using a regularized method.

$$
\hat{\beta}_{(j k)} \leftarrow \operatorname{argmin}_{\beta} \sum_{i}\left(y_{i}-\sum_{j} x_{i(j k)} \beta_{(j k)}\right)^{2}+R .
$$

Calculate the prediction of target values.

$$
\hat{y}_{i} \leftarrow \sum_{j, k} x_{i(j k)} \hat{\beta}_{(j k)}
$$

Fig. 3. Construction of VM model with two-step feature-selection procedure.

Let $X=\left\{x_{i(j k)} \mid 1<i<n, 1<j<p, 1<k<q\right\}$ be the complete dataset of the 
spectroscopic signals. In the first step, each wavelength is summarized by its mean value. The summarized signals $\left(x_{i j}^{\prime}\right)$ are used as predictors to compute the coefficients $\left(\hat{\beta}_{j}\right)$ using a regularized method for selecting significant wavelengths. In the second step, a new dataset is created from the selected wavelengths of the original dataset. The coefficients $\left(\hat{\beta}_{(j k)}\right)$ are calculated by applying a regularized method to the new predictors. The computed coefficients represent the key wavelengths and time points that correspond to the original spectroscopic signals. Finally, the prediction of target values is performed using a linear combination that includes $x_{i(j k)}$ and $\hat{\beta}_{(j k)}$.

\section{Experimental Results}

\subsection{Datasets}

The spectroscopic signals were collected from an optical emission spectroscopy (OES) sensor that measured the chemical changes in a plasma etching process in one of the leading semiconductor companies in South Korea. Fig. 4 illustrates the flow of a plasma etching process used to remove a certain portion of the surface of a semiconductor wafer by a chemical process. The exposed surface is desorbed from the wafer by reaction with a fed gas. This produces a plasma of gas and the OES sensor collects data for the full spectrum of the plasma at frequent time points to capture the plasma state involved in the dynamic reaction. 


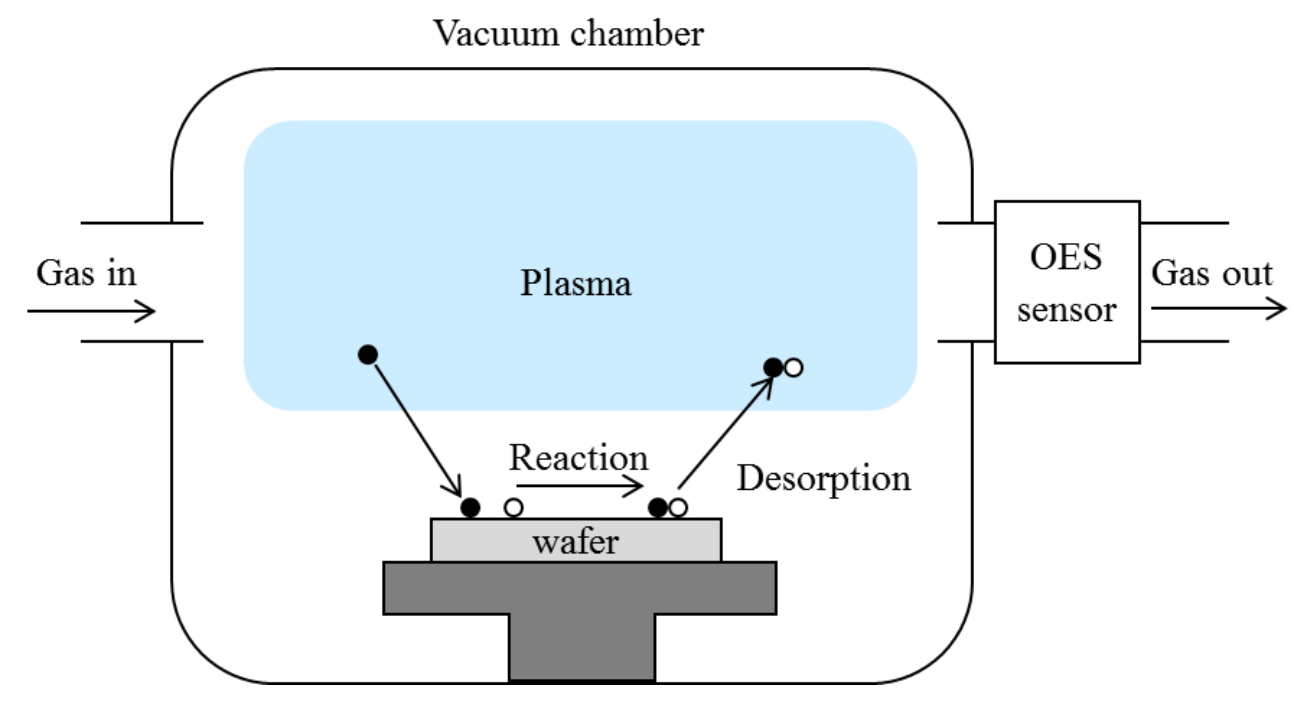

Fig. 4. Flow of a plasma etching process.

To compare the performances of the lasso, elastic-net, and fused lasso-based VM models, we performed experimental studies on real spectroscopic signal data from a semiconductor manufacturing process. Sixty-one samples were collected from three different lots. Each sample consisted of 2,045 wavelength channels containing 160 time samples; hence, the total number of variables in the sample was 327,200.

\section{Table 1}

Training and Testing sets in each case.

\begin{tabular}{ccc}
\hline Case & Training set & Testing set \\
\hline Case 1 & Lot 2, Lot 3 & Lot 1 \\
Case 2 & Lot 1, Lot 3 & Lot 2 \\
Case 3 & Lot 1, Lot 2 & Lot 3 \\
\hline
\end{tabular}


For fair evaluation, we divided the dataset into training and testing sets by lots. Table 1 shows the training and testing sets in each case. The VM prediction model was built from two lots (training sets) and was evaluated from the remaining one lot (testing set).

\subsection{Parameter setting}

Regularized methods increase the generalization capability of the model by penalizing the coefficients. The regularization parameter governs the trade-off between the training accuracy and the generalization accuracy. When the value of the parameter is low, the training accuracy increases; this leads to complex models that tend to select a large number of features and overfit the noise on the dataset. Conversely, large parameter values produce lowcomplexity models that are likely to select a smaller number of features and underfit the dataset. The top panel of Fig. 5 illustrates an example of the number of features selected by the lasso. As the regularization parameter increases, the number of selected features decreases continuously until eventually none are selected. An optimal value of the parameter exists between zero and the maximum value (which forces all coefficients of the model to be zero). To determine the optimal value, we performed five-fold cross-validation, which is a widely used technique for tuning the regularization parameter. 

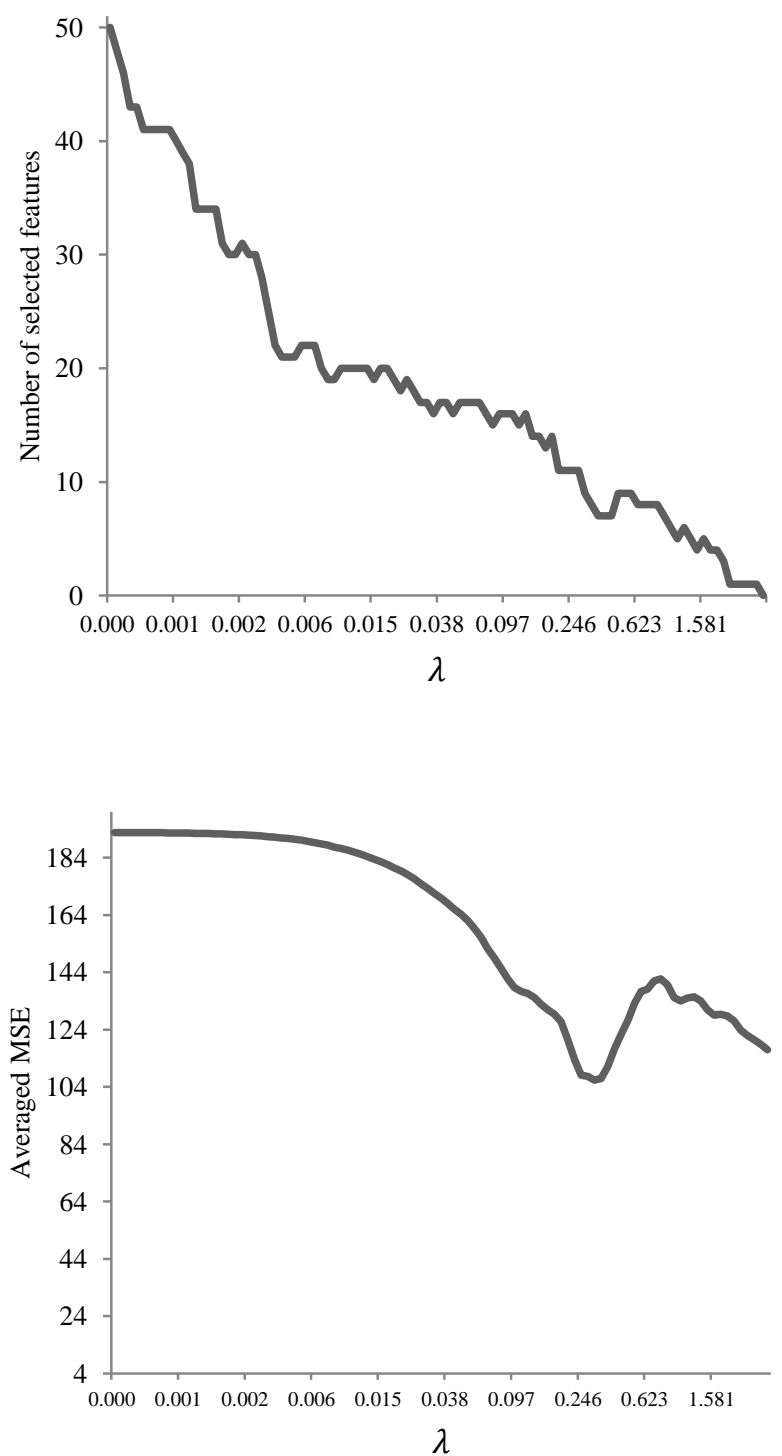

Fig. 5. Number of selected features (top) of the lasso and Averaged MSE (bottom) obtained at different parameter values.

The training dataset was partitioned into five equal-sized subsets. A single subset was retained as the validation set and the remaining four subsets were used to build the model. The single subset was used to assess the quality of the model by calculating the mean squared error (MSE). The cross-validation process was repeated five times and the results were averaged. The bottom panel of Fig. 5 shows an example of the averaged MSE obtained by using this cross-validation technique in the lasso model. We determined the value of $\lambda$ that 
minimizes the averaged MSE. The elastic net and the fused lasso require two penalty

parameters: the lasso penalty parameter and the other. We computed $\lambda_{2}^{\min }$ and $\lambda_{2}^{\max }$ for all $\lambda_{1} \in\left[\lambda_{\min }, \lambda_{\max }\right]$, which can be determined by the lasso model. Within the bounds of $\lambda_{1}$ and $\lambda_{2}$, we determined the parameter that minimizes the averaged MSE. In this study, we used the statistics tool box in MATLAB ver. R2011b for experiments with the lasso and the elastic net. For the fused lasso, the SLEP package [27] in MATLAB ver. R2011b was used.

\subsection{Performance comparison}

In this section, we examine the kind of features selected by the regularization least-squares methods and compare the proposed fused lasso VM model with the lasso and elastic net VM models in terms of prediction accuracy. The top panel of Fig. 6 shows the coefficients obtained by the lasso, which selects only three features. This result illustrates a limitation of the existing lasso algorithm, which is a tendency to select only one feature from a group of highly correlated features. The middle panel of Fig. 6 presents the coefficients defined by the elastic net, which selects several groups of features included in four different wavelengths. However, it does not reflect the grouping effect, in which equal coefficients are given to highly correlated and adjacent features. The bottom panel of Fig. 6 illustrates how the fused lasso selects consecutive features that are correlated. The grouping tendency strongly affects the coefficient, leading to the selection of neighboring features that receive equal coefficients. 

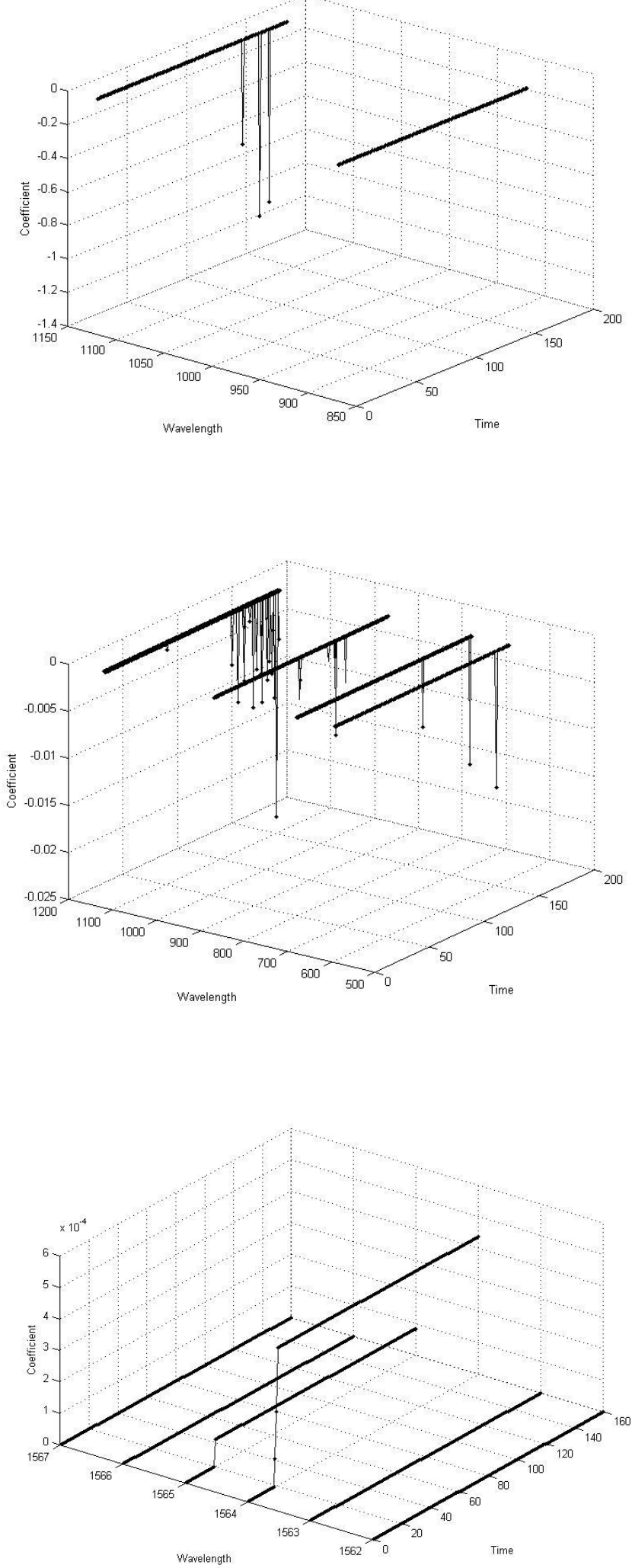

Fig. 6. The coefficients defined by the lasso (top), elastic net (middle), and fused lasso (bottom). 
Stability is an important requisite of a feature-selection algorithm to construct robust prediction models. Small fluctuations in the selected subset of features may occur because the conditions of semiconductor tools can change over time, and noise can exist in the process data collected from the tools. However, the selection of key features should not change considerably because of these reasons. To verify whether the three regularized methods select subsets regularly with variations in the process data, we measured the similarities between the subsets of selected features using the Tanimoto distance, which is calculated as follows [28]:

$$
\text { Similarity }\left(s, s^{\prime}\right)=1-\frac{|s|+\left|s^{\prime}\right|-2\left|s \cap s^{\prime}\right|}{|s|+\left|s^{\prime}\right|-\left|s \cap s^{\prime}\right|},
$$

where $s$ is the subset of features. The Tanimoto similarity measures the amount of overlapping features between two sets of arbitrary cardinalities. The similarity takes values between zero and one, with zero indicating no overlap between the two sets and one indicating that the two sets are identical.

\section{Table 2}

Similarities between the subsets selected from each case.

\begin{tabular}{cccc}
\hline Similarity & Lasso & Elastic net & Fused lasso \\
\hline Similarity $\left(s_{1}, s_{2}\right)$ & 0.35 & 0.18 & 0.81 \\
Similarity $\left(s_{2}, s_{3}\right)$ & 0.20 & 0.20 & 0.81 \\
Similarity $\left(s_{1}, s_{3}\right)$ & 0.00 & 0.86 & 1.00 \\
\hline Average & 0.18 & 0.20 & 0.86 \\
\hline
\end{tabular}

Table 2 shows the Tanimoto similarities between the subsets of features, where $s_{i}$ denotes the subset of selected features from the $i$-th case. The fused lasso model consistently extracts similar subsets of features in all cases, in contrast to the other models. This results from the 
fact that the second penalty term of the fused lasso encourages feature selection to sort successive groups of features that are highly correlated with each other. For sufficiently large regularization parameters, only one group of consecutive features is selected, which rarely fluctuates with variations in the process data.

\section{Table 3}

Comparison of VM estimation performances.

\begin{tabular}{|c|c|c|c|c|c|c|c|c|c|}
\hline \multirow{2}{*}{ Case } & \multicolumn{3}{|c|}{ Lasso } & \multicolumn{3}{|c|}{ Elastic net } & \multicolumn{3}{|c|}{ Fused lasso } \\
\hline & MSE & $\begin{array}{c}\text { MAPE } \\
(\%)\end{array}$ & $\begin{array}{c}\text { Max } \\
\text { Error } \\
(\%)\end{array}$ & MSE & $\begin{array}{c}\text { MAPE } \\
(\%)\end{array}$ & $\begin{array}{c}\text { Max } \\
\text { Error } \\
(\%) \\
\end{array}$ & MSE & $\begin{array}{c}\text { MAPE } \\
(\%)\end{array}$ & $\begin{array}{c}\text { Max } \\
\text { Error } \\
(\%)\end{array}$ \\
\hline Case 1 & 379.73 & 5.20 & 13.79 & 451.01 & 5.43 & 17.22 & 415.16 & 5.24 & 14.95 \\
\hline Case 2 & 163.07 & 3.93 & 11.01 & 207.45 & 4.79 & 9.60 & 86.64 & 2.51 & 7.93 \\
\hline Case 3 & 415.82 & 6.09 & 14.74 & 174.11 & 4.23 & 10.28 & 157.57 & 3.33 & 8.82 \\
\hline Average & 319.54 & 5.07 & 13.18 & 277.52 & 4.82 & 12.36 & 219.79 & 3.70 & 10.56 \\
\hline
\end{tabular}

Table 3 shows a comparison of the VM estimation performances. The prediction performance is expressed in terms of MSE, mean absolute percentage error (MAPE), and maximum error (MaxError) that can be calculated by the following equations:

$$
\begin{aligned}
\text { MSE } & =\frac{1}{N} \sum_{i=1}^{N}\left(y_{i}-\hat{y}_{i}\right)^{2}, \\
\text { MAPE } & =\frac{1}{N} \sum_{i=1}^{N}\left|\frac{y_{i}-\hat{y}_{i}}{y_{i}}\right| \times 100 \%, \\
\text { MaxError } & =\max _{i=1, \ldots, n}\left|\frac{y_{i}-\hat{y}_{i}}{y_{i}}\right| \times 100 \% .
\end{aligned}
$$

where $\hat{y}$ and $y$ are, respectively, the prediction values and the actual metrology values (quality variable). $N$ is the total number of observations. The smaller MSE, MAPE and 
MaxError values indicate better prediction performance of the VM model. Note that the performances of the VM models are reported in the terms of the MSE, MAPE, and MaxError for the test-set prediction of each model based on its selected features. In case 1, the fused lasso shows performance comparable to other models. In cases 2 and 3, all performance measures of the fused lasso are substantially lower than that of the lasso and the elastic net. This implies that the performance of the VM model is influenced by property of the fused lasso. The only difference between the lasso and fused lasso is the second $L_{1}$-penalty, which encourages the selection of adjacent features. Consequently, the $L_{l}$-penalty produces a grouping tendency and a robust subset of features that can properly accommodate datasets with consecutive signals that are correlated, such as that of spectroscopic signals.

\section{Conclusions}

In this paper, we proposed a fused lasso model to identify significant features in spectroscopic signals so as to construct a reliable VM model. Prediction modeling of spectroscopic signals must address two main challenges: (1) the signals involve combinations of multiple samples collected over time, with each sample containing a vast number of features; (2) consecutive features are highly correlated because of the natural order present in the dataset. The first contribution of this work is adapting the feature-selection procedure to handle the problems posed by the complexity of spectroscopic signals, one of which is the enormous number of data points. The overall procedure of the proposed idea is split into two steps: the first to select the important wavelengths, and the second to select the key time points of the wavelengths extracted in the first step. A second contribution of this work is the application of the fused lasso algorithm to the analysis of process control using multivariate statistics, which are especially useful for analyzing high-dimensional data with features that can be ordered in meaningful ways. To the best of our knowledge, this is the first attempt to 
use the fused lasso for purposes of VM.

Our experiments with real spectroscopic signals demonstrate the usefulness and robustness of the fused lasso-based VM model over the existing lasso- and elastic net-based VM models. The fused lasso-based VM model outperformed the other VM models in terms of prediction accuracy, and also showed improved reliability by providing a robust subset of selected features.

This study mainly focuses on verification of the selected features from the computational point of view. That is, the current study compares the performance of feature selection results based on the prediction accuracy with the features selected by each computational algorithm. However, it should be much better if the selected features are confirmed by the physics perspectives as well. This is a clear limitation of the present study. Nevertheless, we believe that the outcomes of the present study will stimulate an ongoing investigation into the development of ever better tools for VM modeling to manufacturing processes.

\section{Acknowledgments}

We thank the editor and referees for their constructive comments and suggestions, which greatly improved the quality of the paper. This work was supported by the BK21 Plus and Basic Science Research Program, through the National Research Foundation of Korea, funded by the Ministry of Science, ICT, and Future Planning (2013007724). This work was conducted during Seoung Bum Kim's visit to DIMACS partially enabled through support from the National Science Foundation under grant numberCCF-1144502.

\section{References}


[1] P. Chen, S. Wu, J. Lin, F. Ko, H. Lo, J. Wang, et al., Virtual metrology: a solution for wafer to wafer advanced process control, in: IEEE Int. Symp. Semicond. Manuf., 2005: pp. 155-157. doi:10.1109/ISSM.2005.1513322.

[2] Y.J. Chang, Y. Kang, C.L. Hsu, C.T. Chang, T.Y. Chan, Virtual metrology technique for semiconductor manufacturing, in: IEEE Int. Jt. Conf. Neural Netw., 2006: pp. 5289-5293. doi:10.1109/IJCNN.2006.247284.

[3] S. Lynn, N. Macgearailt, J. V Ringwood, Real-time virtual metrology and control for plasma etch, J. Process Control. 22 (2012) 666-676. doi:10.1016/j.jprocont.2012.01.012.

[4] P. Prakash, A. Schirru, P. Hung, S. McLoone, MSC-clustering and forward stepwise regression for virtual metrology in highly correlated input spaces, in: Adv. Semicond. Manuf. Conf., 2012: pp. 45-50. doi:10.1109/ASMC.2012.6212866.

[5] T.H. Lin, F.T. Cheng, W.M. Wu, C.A. Kao, A.J. Ye, F.C. Chang, NN-based key-variable selection method for enhancing virtual metrology accuracy, IEEE Trans. Semicond. Manuf. 22 (2009) 204-211. doi:10.1109/TSM.2008.2011185.

[6] Y.T. Huang, F.T. Cheng, Y.T. Chen, Importance of data quality in virtual metrology, in: Ind. Electron. Conf., 2006: pp. 3727-3732. doi:10.1109/IECON.2006.347318.

[7] L. Xu, W.J. Zhang, Comparison of different methods for variable selection, Anal. Chim. Acta. 446 (2001) 475-481. doi: 10.1016/S0003-2670(01)01271-5.

[9] J. Wan, B. Honari, M. Sean, A dynamic sampling methodology for plasma etch processes using Gaussian process regression, in: Information, Commun. Autom. Technol., 2013: pp. 16.

[10] S. Park, S. Jeong, Y. Jang, S. Ryu, H. Roh, G. Kim, Enhancement of the Virtual 
Metrology Performance for Plasma-Assisted Oxide Etching Processes by Using Plasma Information ( PI ) Parameters, IEEE Trans. Semicond. Manuf. 28 (2015) 241-246. doi: 10.1109/TSM.2015.2432576

[11] T. Hirai, M. Kano, Adaptive Virtual Metrology Design for Semiconductor Dry Etching Process Through Locally Weighted Partial Least Squares, IEEE Trans. Semicond. Manuf. 28 (2015) 137-144. doi: 10.1109/TSM.2015.2409299.

[12] J. Liu, Developing a soft sensor based on sparse partial least squares with variable selection, J. Process Control. 24 (2014) 1046-1056. doi:10.1016/j.jprocont.2014.05.014.

[13] Z.X. Wang, Q.P. He, J. Wang, Comparison of variable selection methods for PLS-based soft sensor modeling, J. Process Control. 26 (2015) 56-72. doi:10.1016/j.jprocont.2015.01.003.

[14] C. Shang, X. Huang, J.A.K. Suykens, D. Huang, Enhancing dynamic soft sensors based on DPLS: A temporal smoothness regularization approach, J. Process Control. 28 (2015) 1726. doi:10.1016/j.jprocont.2015.02.006.

[15] A. Schirru, S. Pampuri, C. De Luca, Multilevel Kernel Methods for Virtual Metrology in Semiconductor Manufacturing, in: IFAC World Congr., 2011: pp. 11614-11621.

[16] R. Tibshirani, Regression shrinkage and selection via the lasso, J. R. Stat. Soc. Ser. B Stat. Methodol. 58 (1996) 267-288.

[17] S. Pampuri, A. Schirru, G. Fazio, G. De Nicolao, Multilevel Lasso applied to virtual metrology in semiconductor manufacturing, in: IEEE Int. Conf. Autom. Sci. Eng., 2011: pp. 244-249. doi:10.1109/CASE.2011.6042425.

[18] H. Zou, T. Hastie, Regularization and variable selection via the elastic-net, J. R. Stat. Soc. 
67 (2005) 301-320.

[19] G.A. Susto, A.B. Johnston, P.G. O’Hara, S. McLoone, Virtual metrology enabled early stage prediction for enhanced control of multi-stage fabrication processes, in: IEEE Int. Conf. Autom. Sci. Eng., 2013: pp. 201-206. doi:10.1109/CoASE.2013.6653980.

[20] R. Tibshirani, M. Saunders, S. Rosset, J. Zhu, K. Knight, Sparsity and smoothness via the fused lasso, J. R. Stat. Soc. Ser. B Stat. Methodol. 67 (2005) 91-108. doi:10.1111/j.14679868.2005.00490.x.

[21] E. Ragnoli, S. McLoone, S. Lynn, J. Ringwood, N. Macgearailt, Identifying key process characteristics and predicting etch rate from high-dimension datasets, in: Adv. Semicond. Manuf. Conf., 2009: pp. 106-111. doi:10.1109/ASMC.2009.5155966.

[22] J. Yang, C. McArdle, S. Daniels, Dimension reduction of multivariable optical emission spectrometer datasets for industrial plasma processes, Sensors. 14 (2013) 52-67. doi: $10.3390 / \mathrm{s} 140100052$.

[23] X. Yan, Modified nonlinear generalized ridge regression and its application to develop naphtha cut point soft sensor, Comput. Chem. Eng. Chem. Eng. 32 (2008) 608-621. doi:10.1016/j.compchemeng.2007.04.011.

[24] T. Hastie, R. Tibshirani, J. Friedman, The elements of statistical learning: data mining, inference and prediction, 2009. doi: 10.1007/BF02985802.

[25] D. Zeng, C.J. Spanos, Virtual metrology modeling for plasma etch operations, IEEE Trans. Semicond. Manuf. 22 (2009) 419-431. doi:10.1109/TSM.2009.2031750.

[26] B. Ma, S. McLoone, J. Ringwood, N. Macgearailt, Selecting signature optical emission spectroscopy variables using sparse principal component analysis, in: Int. Conf. Comput. Inf. 
Technol., 2008: pp. 14-19. doi:10.1109/ICCITECHN.2008.4803104.

[27] J. Liu, S. Ji, J. Ye, SLEP: Sparse learning with efficient projections, Arizona State Univ. (2009). http://www.public.asu.edu/ jye02/Software/SLEP/manual.pdf.

[28] A. Kalousis, J. Prados, M. Hilario, Stability of feature selection algorithms: A study on high-dimensional spaces, Knowl. Inf. Syst. 12 (2007) 95-116. doi: 10.1007/s10115-0060040-8.

[29] H. Hoefling, A Path Algorithm for the Fused Lasso Signal Approximator, J. Comput. Graph. Stat. 19 (2010) 984-1006. doi:10.1198/jcgs.2010.09208.

[30] S. Pampuri, A. Schirru, G.A. Susto, C. De Luca, A. Beghi, G. De Nicolao, Multistep Virtual Metrology Approaches for Semiconductor Manufacturing Processes, in: IEEE Int. Conf. Autom. Sci. Eng., 2012: pp. 91-96. 